\title{
TRES ESTALLIDOS EN EL HORIZONTE LITERARIO DE LA MODERNIDAD Y LA POSMODERNIDAD (Notas sobre Jakobson, Sterne, la mujer invisible y el diccionario de Godard) '
}

Three Outbreaks on the Literary Horizon of Modernity and Postmodernity

(Notes on Jacobson, Sterne, The Invisible Woman and Godard's Dictionary)

\author{
JUAN CARLOS RODRÍGUEZ \\ Universidad de Granada \\ España \\ jcrodri@ugr.es
}

(Recibido 3O-II-2OI4; aceptado I6-I2-2OI4)

I.- Comenzaré de forma un tanto abrupta pues ya iremos desgranando las cuestiones que aquí esquematizo. Diré así en primer lugar, que considero que la estética es una de las maneras de configurar la imagen del "yo-soy-libre porque he nacido libre por naturaleza”. Que es a su vez el eje del inconsciente ideológico/ libidinal del capitalismo. Pues no olvidemos que por un lado están las relaciones socio-vitales existentes y por otro lado la forma en que esas relaciones sociales nos configuran: configuran nuestra manera de vivirlas, de pensarlas, de experienciarlas; nuestra forma de concebir tales realidades vitales. A eso lo llamo nuestro inconsciente ideológico/ libidinal. No sin problemas o enigmas. En el ámbito literario el enigma de la poesía ha solido centrar la mayoría de los debates. Como no sé la diferencia que puede haber entre modernidad y posmodernidad, aludiré a esos términos como apoyos cómodos cuando los necesite. Señalando desde ahora que tales términos únicamente tienen sentido -acaso- a propósito de las lógicas variaciones históricas de un continuum capitalista cuyo fondo de explotación de las vidas no ha cambiado sin embargo nunca. A esas lógicas variantes históricas es a lo que llamo la radical historicidad de la literatura. Puesto que la ideología inconsciente jamás es homogénea sino que está llena de brechas o fisuras he denominado estallido a algunas de sus contradicciones más profundas.

\footnotetext{
I Para citar este artículo: Rodríguez, Juan Carlos (20I4). Tres estallidos en el horizonte literario de la modernidad y la posmodernidad). Alabe 10. [www.revistaalabe.com]

DOI Io.I5645/Alabe.2OI4.IO.IO
} 
2.- El primer estallido o la primera gran contradicción la centraré, pues, en torno al aludido enigma de la poesía. Y podemos fijarnos en una fecha que considero bien sintomática: el año r958. ¿Qué significado podemos extraerle a esa fecha? Para el terreno que ahora nos interesa, es decir, las relaciones dentro del ámbito literario y su inscripción en el inconsciente del "yo soy libre”, supongo que podríamos extraer múltiples imágenes a propósito de este primer estallido.

Pues veamos. En I958 se celebró en Bloomington, en la Universidad de Indiana, un célebre congreso sobre lingüística y literatura. No nos olvidemos de una cuestión básica: Estados Unidos era prácticamente un páramo en el ámbito de la teoría y la crítica literaria. Tampoco es que en ese terreno hubiéramos ido mucho más allá en la Europa Occidental (y menos en la España de Franco). Aunque en Europa y en España hubiéramos vivido, especialmente en los años 20 y 30 , todos los avatares de las vanguardias. Y eso ya desde la bisagra del XIX-XX y, sobre todo, antes y después de la Primera Guerra Mundial. Recordemos sólo el modernismo de Darío, el “noucentisme” catalán, las llamadas generaciones del I 4 y del 27, el cubismo, el dadaísmo y el surrealismo; y con tremenda fuerza el debate entre la pureza y el compromiso en la II República y en toda Europa. Pues bien: aunque conociéramos además la estética de Croce y Gramsci e igualmente el horizonte fenomenológico desde Husserl a Heidegger, y desde Merleau-Ponty a las ciencias de la cultura y de las formas simbólicas en Dilthey y en Cassirer, etc., aunque todo eso hubiera sido así, seguíamos sin duda en las nubes respecto a la poesía. Y repito que más se diluía el asunto en los propios USA, pese a que ya existiera el New Criticism y pese a que Wellek y Warren hubieran publicado su Teoría de la literatura en i949. La verdad era que al hablar de la poesía seguíamos moviéndonos entre el "poesía eres tú" y el horizonte de lo inefable. Tanto es así que, en I948, cuando Sartre publica la primera edición de ¿Qué es la literatura? y saca a relucir toda la avalancha del compromiso (con indudables raíces en la Resistencia contra los nazis), Sartre sin embargo excluye a la llamada poesía lírica de tal compromiso, puesto que en el fondo la poesía seguía siendo algo propio sólo de la intimidad del alma, etc.,

Volvamos, así, ahora al Congreso de la Universidad de Indiana, o mejor dicho, a la mil veces reeditada, comentada y condensada "Conferencia de clausura". Es bien sabido que esa "conferencia de clausura" estuvo a cargo de un ruso "bueno" (pues vivíamos en plena Guerra fría desde i947-48), un ruso que, tras formarse con los Formalistas de su país y luego con los Funcionalistas checos, se había trasladado a Estados Unidos donde fue adquiriendo un gran renombre como lingüista. Durante la II Guerra mundial ese ruso había coincidido además en Nueva York con un joven Lévi-Strauss, con el que trabó una profunda amistad que posteriormente se plasmaría por ejemplo en el análisis conjunto del soneto Los gatos, de Baudelaire, etc.

Y a lo que íbamos: mientras por nuestra parte seguíamos con el "poesía eres tú" o "poesía es lo inefable", ese ruso, o sea, Roman Jakobson, clausuraba el congreso de Indiana más o menos así: ¿Queréis saber lo que es poesía? Pues ahí va, os lo voy a decir. Y lo dijo de este modo: poesía (o función poética, da igual) es simplemente esto: 


\section{La proyección del principio de equivalencia del eje de la selección al eje de la combinación.}

Nada menos. Hay que imaginarse por un momento la reacción del auditorio. Primero estupor y asombro. Y enseguida el alborozo general: la retórica de Jakobson había cumplido perfectamente su finalidad persuasiva. Gracias a esa conferencia (que, como se sabe, Jakobson tituló Lingüística y Poética²) al fin teníamos una definición de la poesía en términos tan técnicos y objetivos como pudiera exigir el mayor rigor científico (pues no olvidemos tampoco que estábamos, y estamos, en plena hegemonía del tecnicismo cientifista). Claro que tras el primer asombro vino la reflexión y la conmoción del estallido comenzó a desinflarse. En cuanto la fórmula se tradujo al "roman paladino" se la desmenuzó poco a poco: Jakobson en realidad no se refería más que a lo que los primeros re-descubridores de Saussure llamaban paradigma y sintagma, algo que él confirmó sin problemas. A lo que se había referido era al juego de las metáforas (el eje de la semejanza) y al de las metonimias (eje de la contigüidad) dentro del texto poético. Y a la vez a las equivalencias o al paralelismo entre elementos fonéticos, sintácticos y semánticos en el interior del poema. En suma, a la atención puesta en el "mensaje en sí”, o sea, a los propios signos y no a su exterior. Todo lo que se resumía en una sola afirmación: que lo que se expresaba en la poesía era la intimidad del lenguaje.

No es que el trabajo de hecho de Jakobson hubiera resultado en vano, es que aquella fórmula tan aparentemente científica y técnica se iba desvaneciendo, puesto que a fin de cuentas se inscribía en el mismo horizonte ideológico de toda la crítica fenomenológica: buscar el "en sí" del lenguaje era buscar la pureza del lenguaje como plasmación de la intimidad del alma, como plasmación del yo-soy-libre más puro. Con lo cual en verdad estábamos en el mismo lugar del que habíamos partido. Incluso hubo más críticas: no sólo es que Jakobson no explicara por qué un poema podía considerarse bueno o malo; no sólo es que hubiera dejado de lado la prosa, considerándola simplemente como "a medio camino" entre la función poética y la función referencial (algo que obviamente no indicaba nada); es que había dejado de lado la Historia: para Jakobson la poesía había vivido en un presente perpetuo, una sincronía eterna, un universal inalterable. Desde los que suelen llamarse cantos heroicos hindúes a los sonetos de Shakespeare y hasta Pushkin, Yeats o Valéry, todo habría sido lo mismo: un "uso magistral" de los términos del lenguaje por parte de un poeta libre y puro. Y para ese viaje no se necesitaban tantas alforjas. Incluso Jakobson llegaba a ampararse en el propio Valéry: la afirmación de este de que la poesía era "una vacilación entre sonido y sentido" le parecía a Jakobson más científica que cualquier otra.

\footnotetext{
${ }^{2}$ Las intervenciones en este congreso se publicaron dos años después al cuidado de Thomas Sebeok con el título de Style in Language, Cambridge, Mass., I96o. La versión española de la conferencia de Jakobson "Lingüística y poética" puede verse en Ensayos de lingüistica general, trad. de J. M. Puyol y J. Cabanes, ed. Seix-Barral, Barcelona, I975, pp. 347-365.
} 
Lo cual provocaba más contradicciones en torno al estallido de Jakobson y sus consecuencias posteriores. Puesto que apenas unos años después, en I966, en otro congreso célebre (ahora en la John Hopkins University) Derrida presentó su famosa ponencia: Estructura, Signo y Representación (o Juego) en el discurso de las Ciencias Humanas, que supuso el comienzo de lo que iba a ser el auge avasallador de la De-Construcción en los Usa. Y ello en competencia con el Generativismo de Chomsky y con toda la ideología teórica de la Comunicación y la Semiótica textual. En suma, y como diría Richard Rorty, se imponía el Giro lingüístico: no el análisis de las ideas sino de los enunciados o proposiciones; no analizar sólo el mundo del texto sino considerar al mundo como texto. Es decir, el triunfo absoluto del capitalismo ideológico más descarnado. Pues fijémonos en que en el mismo I95 ${ }^{8}$-o quizás un poco antes- la Alemania de Hitler (reconvertida en la Alemania de Adenauer), la Italia de Mussolini (gobernada por la Democrazia cristiana heredera de De Gasperi) y la Francia de Vichy (ahora de De Gaulle), o sea, las tres potencias nazis-fascistas derrotadas en la Segunda Guerra mundial firmaban en Roma el primer tratado de la Unión Europea. Bajo el manto protector de los Usa, en apenas unos diez años y gracias al Plan Marshall, todos los nazi-fascistas europeos se habían convertido en demócratas y exhibían la libertad como bandera, puesto que la recuperación económica parecía asombrosa: se habló así del "milagro alemán” y -con mayor ironía, claro- del "miracolino italiano". Menos la España de Franco, todo el occidente europeo se había acostado fascista y se había despertado democristiano (o social-demócrata). Por supuesto que tampoco había por qué asombrarse demasiado: la infraestructura capitalista seguía siendo la misma; sólo había que americanizar los gestos y los gustos y los estilos de vida y de conducta parlamentaria, etc. Únicamente el Reino Unido era un caso aparte, y a Inglaterra volveremos para hablar de nuestro segundo estallido.

3.- Segundo estallido: obviamente la Ilustración europea del XVIII sí que es el verdadero arranque de la Modernidad. Pero además, en el siglo XVIII, Inglaterra era ya la "más burguesa de las naciones", en frase lapidaria de Marx. No sólo porque en i649 el Parlamento, con Cromwell a la cabeza, hubiera decapitado al rey Carlos I; no sólo por la llamada "revolución gloriosa" de i688 (el Parlamento imponiéndose definitivamente a la Monarquía, aunque conservándola), ni siquiera por su potencial marítimo y el valor de sus mercados nacionales e internacionales (lo que culminaría con la revolución industrial y el gran Imperio británico del XIX). No sólo por eso, digo, sino por la conmoción que todo eso había provocado en el nivel básico de las relaciones socio-vitales cotidianas. En un esquema muy simple: las relaciones familiaristas burguesas se habían impuesto sobre el linaje de la sangre azul nobiliaria. Una aristocracia que por supuesto seguiría existiendo hasta hoy, con sus ritos y sus parafernalias perviviendo en todos los terrenos. Sólo que con un matiz decisivo: la propia aristocracia se había reconvertido ya introduciéndose en el ámbito capitalista. De modo que puede decirse que la revolución burguesa estaba ya establecida en gran manera en Inglaterra (o Gran Bretaña tras la unión con Escocia: Irlanda sería siempre una colonia católica, rural y retrógrada en el imaginario británico). 
Pues bien, nuestro segundo estallido lo vamos a centrar en una novela como el Tristram Shandy y un escritor que por casualidad nació sin embargo en Irlanda (son innumerables los escritores irlandeses que posiblemente hayan escrito el mejor inglés literario, desde Swift a James Joyce). Y digo que Laurence Sterne (o sea, nuestro escritor) nació por casualidad en Irlanda pues era hijo de un alférez o abanderado de un destacamento inglés allí establecido. Pero en verdad Sterne creció y se formó no sólo en una Inglaterra protestante etc., sino sobre todo en ese humus fermentador del familiarismo burgués al que habíamos aludido. Aunque se tenía un tremendo respeto por el lenguaje y el gusto aristocrático sobre todo en el terreno artístico. Hume dedicará toda su vida a trasladar ese gusto aristocrático a la clase media para conseguir al menos que esa burguesía inglesa no tuviera -dice Hume, copiando literalmente a Rousseau- el lenguaje, el gusto y los modales tan rudos "como los de un suizo educado en Holanda".

Pero lo que nos importa: ya que hablamos del familiarismo burgués, deberíamos señalar que el matrimonio del clérigo Sterne fue más bien un desastre. No sólo porque este párroco de York, comido por la tuberculosis, fuera un juerguista empedernido, sobre todo en las fiestas de señoras y señores que se celebraban en el castillo (que ellos llamaban Crazy Castle) que había heredado su amigo John Hall-Steveson (Eugenius, en el Tristram), con resacas tremendas que Sterne procuraba curarse tomando la famosa agua negra de alquitrán que había recomendado el obispo y filósofo Berkeley. No sólo por esto, digo, sino porque su mujer, Elizabeth Lumley, con la que tuvo a su hija Lydia (que luego le serviría de amanuense), su mujer, digo, sufría auténticos delirios mentales en los que se consideraba Reina de Bohemia ("El rey de Bohemia y sus siete castillos” será un relato que aparece en el libro. Aunque Bohemia era también un bosque cercano a su casa). Pero en I76o, a los 46 años, la vida de Sterne dio un vuelco inesperado.

Había llevado al librero londinense Dodley los dos primeros volúmenes del Tristram que se publicaron en enero del 6o. Se dice que fue la sensación de ese Año Nuevo. En York se vendieron cien ejemplares diarios y en Londres la edición se agotó de inmediato, tanto que en Abril hubo que sacar a la luz una segunda edición de este "libro de Yorick", que era como se le conocía. Por supuesto Yorick es una creación a medias entre Shakespeare y Cervantes: es el bufón de la calavera con la que Hamlet medita y es a la vez un personaje cervantino que cabalga sobre un rocín como Rocinante y que como Cervantes ironiza siempre sobre todas las cosas. Curiosamente el nombre de Shakespeare no aparece en el libro y el de Cervantes aparece continuamente. Y Yorick desde luego es el "alter ego" de Sterne tanto en el Tristram como en su otro libro El viaje sentimental.

Pero vamos a aclararnos. Sterne nos dice que va a contar la vida y las opiniones de Tristram Shandy, gentleman. Sin embargo cuando acaba el segundo volumen Tristram no ha nacido todavía. La voz del propio protagonista, o sea, Tristram, nos lo dice en el capí-

\footnotetext{
3 Vid L. Sterne: The Life and Opinions of Tristram Shandy, Gentlman, Penguin Books, I967. La magnífica traducción Española de Javier Marías, ahora en: Tristram Shandy, Alfaguara, Madrid, 2OII, con introducción de Andrew Wright. Marías llegó a decir que esa era su “mejor obra”.
} 
tulo XIV del primer volumen: "En suma, es el cuento de nunca acabar;-por mi parte les aseguro que estoy en ello desde hace seis semanas, yendo a la mayor velocidad pasible- $y$ no he nacido aún". Más ironía, imposible. Pero es que un poco más abajo nos añade que va a seguir contándonos su vida publicando dos volúmenes al año. Y así sucedió en efecto desde I76o hasta I766. Veamos un esquema mínimo de lo que ocurre en este libro.

Porque lo que sucede es que no sucede nada. De la vida de Tristram sólo conoceremos tres o cuatro cosas que le acaecen hasta los cinco años. Son las opiniones de Tristram, o sea la voz que le presta Sterne, lo único que realmente constituye la urdimbre de los nueve volúmenes del libro. Pero qué opiniones y qué estructura: digresiones, divagaciones, asteriscos, guiones cortos o larguísimos, desorden en la numeración de los capítulos, capítulos largos o de una sola frase, lógica causal completamente interrumpida, páginas en blanco o en negro (cuando mata a Yorick en el primer libro le dedica dos páginas en negro por luto, y luego lo resucita, claro); páginas rayadas, temas dejados sueltos y retomados más tarde de manera inesperada y además con otro asunto y con otro sentido... Es una prosa única, rellena de ambigüedad en cada línea: cada palabra, cada frase, cada guión o asterisco quiere decir una cosa y a la vez su contraria o sus posibles ramificaciones hasta el infinito.

En el volumen I y II Tristram nos cuenta su engendramiento. Esta historia es bien conocida. Su padre, el señor Shandy, el primer domingo de cada mes, cumplía con tres obligaciones: ir a la iglesia por la mañana y, luego por la noche, darle cuerda al gran reloj de la casa y procurar engendrar un hijo con la señora Sandhy. Este ritual había creado en la mente de la señora Shandy una asociación de ideas inevitable. Pero la noche del engendramiento algo falla en tal asociación de ideas: de modo que cuando el señor y la señora Shandy están cumpliendo con la obligación de engendrar un hijo, en el momento álgido, la señora Shandy interrumpe a su marido y le pregunta: "Perdona, querido, ¿no te has olvidado de darle cuerda al reloj?”. Obviamente el señor Shandy se altera de manera inevitable y exclama: "A qué mujer se le ocurriría interrumpirme precisamente en este momento". Pues por culpa de la interrupción apenas deposita una débil semilla hacia el útero de la señora Shandy. Pero lo estoy contando mal: no se trataba de una semilla, sino de un homúnculo. Pues como sabremos, tal interrupción no dejó que el homúnculo pudiera ir con suficiente vitalidad y fuerza.

Obviamente Sterne se está burlando de la "embriología” o la "espermática” del machismo remotamente aristotélico aún vigente en su época. Se suponía que el esperma masculino portaba ya en sí un homúnculo, una especie de hombrecillo ya hecho en miniatura, que habría luego de calentarse y vitalizarse en el vientre de la madre. De modo que Tristram, el homúnculo, nos contará su vida y sus opiniones desde ese vientre materno.

Pero fijémonos: si los dos primeros volúmenes (apenas cien páginas) están dedicados al engendramiento de Tristram, el tres y el cuatro se dedican al parto y al nombre del homúnculo. El señor y la señora Shandy habían tenido una disputa: ella quería parir en Londres, donde todo era mejor y más seguro. El señor Shandy se impone, como es lógico: debe parir en su casa, en el pueblo. Sólo que allí sólo hay una comadrona y un mé- 
dico partero y papista, el doctor Slop. Mientras el parto sucede lentísimo en la habitación de arriba, en el salón de abajo, el señor Shandy, el Tío Toby, su ayudante el cabo Trim y el médico partero y papista hablan interminablemente sobre la cesárea, el forceps (que el médico aduce como un nuevo gran invento) y, sobre todo, sobre el bautismo. La burla descarnada se centra ahora en el catolicismo, sacando a colación un texto francés -auténtico- acerca del bautismo del homúnculo introduciendo una cánula en el vientre de la madre e incluso (¿por qué no?) bautizar a todos los homúnculos posibles introduciendo esa cánula en los genitales del padre. La criada Susanah avisa de que el parto es inminente y el forceps del doctor -se nos cuenta luego- aplasta la nariz del niño. El señor Shandy achaca eso a la mala suerte de que el niño naciera por la cabeza y no por los pies -como debería ser, afirma-.

Pero mucho ojo: Sterne nos dice que cuando habla de nariz habla de nariz aplastada y no de que al niño le aplastaran otra cosa, como ustedes, lectores, estarán pensando -añade-. Las digresiones sobre la nariz y el pene -incluso sobre los bigotes- se vuelven de nuevo irresistibles y por supuesto con una continua connotación sexual. Pero no acaban ahí las desgracias. El padre quiere bautizar a su hijo con el nombre de Trismegisto, nada menos, pero la criada Susanah no entiende bien el nombre y el niño acaba siendo bautizado como Tristram.

Es obvio que hoy, en el inglés actual, las correlaciones latinas entre Tristram y tristeza o desgracia (como los Tristia de Ovidio) no se aprecian de hecho, pero en el XVIII era algo mucho más claro, y sobre todo para un clérigo licenciado en Cambridge como Sterne. Los volúmenes 5 y 6 nos muestran a Mister Shandy escribiendo una Tristrapaedia, o sea, una forma de educar a su hijo Tristram. Sólo que divaga tanto que el niño va creciendo más aprisa que el tratado del padre. Y así a los cinco años presenciamos la última desgracia de Tristram. Ya hemos visto la desgracia de su engendramiento, la desgracia de su nariz y de su nombre y ahora se nos ofrecerá la última desgracia que presenciaremos, pues a Tristram ya no lo volveremos a ver. Con cinco años, repito, el niño tiene un día ganas de hacer pis. La joven Susanah, convertida en su niñera, siente escrúpulos de cogerle el pene para ayudarle a orinar y le dice si el señorito podría hacerlo solo. Tristram está de pie encima de una ventana y cuando se saca el pene le cae encima la parte de arriba de la ventana-que era de las de guillotina-. No sabemos muy bien lo que ocurre en el jaleo tremendo que se arma, pero el doctor Slop nos dice que todo se arreglará con una simple fimosis. Insisto que de la vida de Tristram ya no sabremos más. Pero sí de sus opiniones o las de Sterne y de los viajes de este por Francia y por Italia, que luego nos contará más detenidamente en El Viaje sentimental a través de Francia e Italia (la parte italiana no la pudo ya redactar)4.

En I766 Sterne publica un último volumen, el IX, que acaba con un chiste imprevisto sobre la famosa historia de una polla y un toro (a cock and a bull). Una Fábula de

\footnotetext{
${ }^{4} \mathrm{Si}$, como es lógico en un libro tan amplio, el Tristram decae algo en los Vols. Vy VI, para tomar vuelo otra vez en los siguientes con los "escarceos amorosos" del Tío Toby y la viuda Wadman, sin embargo El viaje sentimental se suele considerar "perfecto". Vid. lo que digo sobre esta pequeña obra maestra en mi libro La literatura del pobre, ed. Comares, Col. "De guante blanco", Granada, 2OOI, pp. 38I-382 (Ia ed. 1994).
} 
las mejores que en su género "yo he oído jamás”, nos dice Sterne. Se dice que el toro, que era del señor Shandy, pero que prestaba servicio a toda la comunidad cubriendo a las vacas, ya no sirve para eso. El señor Shandy se indigna y proclama que su toro hubiera servido para raptar a la ninfa Europa e incluso que el hijo del criado Obadian era hijo del toro. La señora Shandy dice que no entiende nada sobre esa fábula, y quizá nosotros sólo podamos añadir en verdad que condensa todo lo que nos quería decir el libro: un sinsentido absoluto.

Y acaso pudiera entenderse así si no fuera porque en el capítulo II del segundo volumen hay una alusión directa al Ensayo sobre el entendimiento humano de Locke. Y Sterne nos dice que ese es un libro de historia. Pero mucho ojo de nuevo: "la historia de lo que sucede en la mente de un hombre". La definición es magistral. Pues efectivamente a partir de aquí podemos leer acaso otro anverso del libro, otro tipo de urdimbre que no es distinta pero que sí le añade más complejidad de perspectivas. Trataremos de describirlo adentrándonos con mayor densidad en la Ilustración británica y continental europea.

4.- Intentaremos, pues, precisar más las cosas dando un pequeño rodeo: se insiste mucho en la afirmación de que la llamada estética posmoderna se deriva de la supuesta crisis de la lingüisticidad y de la identidad en nuestros días. Algo que habrían tratado de evitar los textos teóricos tanto de Kant como de Hegel, que, por otra parte, jamás pensaron delimitar unos planteamientos estéticos hasta un momento determinado de sus vidas: hasta tropezarse con el cuerpo. De ahí que Terry Eagleton hable de la Estética como discurso del cuerpo (sin duda bajo la influencia de la teórica del feminismo Toril Moi, su compañera por entonces y a quien está dedicado el libro La estética como ideología); y Rancière habla del paradigma estético como sensorium común (o sea, en relación con la política) donde se distribuyen las cosas y se hace visible el Arte. Pero evidentemente hay que negar que el arte sea "tonto" o "sublime", como quizás podría derivarse de las afirmaciones de Kant y Hegel al respecto. Kant con su juego entre las categorías del entendimiento y las formas de la sensibilidad (o la finalidad sin fin, o sea, fuera de toda inteligencia) y Hegel definiendo la estética como encarnación de la Idea en lo sensible (el juego entre espíritu y materia, etc.), anulando finalmente a la estética misma.

Sólo que respecto al empirismo inglés del que venimos hablando, también me gustaría matizar algo más: por ejemplo en su aludido y obviamente decisivo Ensayo sobre el entendimiento humano John Locke, tanto en la primera edición de i69o como en la sexta y póstuma de izıo, que es la básica, Locke, digo, finaliza su texto con una división de las ciencias en tres clases: Physiké (escrito en griego), o filosofía natural, que trata de los cuerpos y los espíritus (incluso de Dios y los ángeles); Practiké(también en griego) o sea, la regla que rige nuestra conducta hacia la felicidad; y finalmente -y aquí lo inesperado-Semeiotiké, o sea, la ciencia que estudia los signos y las ideas.

Permítanseme dos observaciones. Primera: ¿por qué aparece Dios dentro de la "filosofía natural" de Locke? Sencillamente porque a Locke le resulta imprescindible para legitimar la propiedad privada y el juramento de los contratos que debe ser sagra- 
do. Por eso en sus Cartas sobre la tolerancia (I689), Locke señala que cualquier hombre puede elegir la religión que quiera o no elegir ninguna, sin que nadie le moleste por ello. Pero sin embargo excluye a los papistas y a los ateos. A los papistas porque son súbditos de otro Estado (el Pontificio) y a los ateos porque uno no se puede fiar de ellos en sus juramentos sobre los contratos de propiedad (lo único sagrado para Locke). Y una segunda observación: Locke dice (en la parte tercera de su Ensayo, la dedicada a las palabras) que las palabras son signos sensibles de nuestras ideas y a la vez que la significación de las palabras o de los signos es completamente arbitraria. Y por eso es necesario un lenguaje preciso y exacto. La sombra que hay por debajo no es la arbitrariedad, obviamente; la sombra que hay por debajo es el hecho de la imagen de las palabras como signos sensibles de las ideas. Y digo que es una sombra puesto que ya señalábamos que el giro lingüístico anglosajón (como lo llamó Rorty) consistirá básicamente en borrar las ideas y analizar sólo los enunciados o las proposiciones lingüísticas coherentes, etc.

O sea, que podemos apreciar desde hoy que ya en Locke existe una crisis de la imagen de los signos que evidentemente irá perdurando hasta nuestros días. Pero es que Sterne se ríe de que el lenguaje tenga que ser exacto y preciso: cada una de sus líneas rebosa ambigüedad, como decíamos, y disloca cualquier sentido posible.

Respecto a la crisis de la identidad (o sea del yo-soy-libre: Locke es obviamente el padre del liberalismo, aunque no del llamado "neo-liberalismo" actual) ocurre algo semejante: Locke legitima la identidad, configurándola a través de la memoria, el entendimiento y la voluntad. ¿Pero qué son estos tres términos? Evidentemente para los escolásticos "pre y postridentinos" (los seguidores del papado romano) memoria, entendimiento y voluntad son las tres potencias del alma divina inscrita en cada hombre. Y Locke necesita quitarse eso de en medio porque necesita al individuo libre y autónomo para que su sistema funcione (y toda la ideología capitalista necesita del individuo supuestamente libre y autónomo para poder explotar "libremente", para poder funcionar también).

Claro que el yo psíquico de Locke va directamente contra esas potencias del alma que Descartes había convertido en sustancias. Pero a la vez, Hume negará la división cuerpo/ espíritu: por ejemplo recordando el ingenio de Cervantes en el capítulo XIII del Segundo Quijote, cuando Sancho se alaba por ser buen catador de vinos. En efecto, añade Hume, si el paladar no está refinado, la mente tampoco podrá estarlo. Y así Hume intentará hacer añicos al yo de Locke, precisamente para salvar al yo de otra manera. Estableciendo no una mente previa, como una lámina de cera donde van grabándose las impresiones sensibles para convertirse en ideas, sino como un proceso donde el yo se va a ir constituyendo: el problema de la identidad es obsesivo en Hume, como se sabe, pues en su Tratado sobre la Naturaleza Humana, llega a decir que el yo no existe. O sea, ese yo sólido y previo del que hablaba Locke.

También se suele decir generalmente que Kant hizo una síntesis magistral de Locke y de Hume: pero de nuevo convendría matizar esto, diciendo que Kant los manipula para conducirlos a su propio interés. 
Fijémonos: Kant estaba tan feliz con la primera edición de la impresionante Crítica de la Razón Pura cuando leyó un furibundo ataque contra Hume traducido del inglés al alemán. Y Kant se asustó tanto con lo que leyó ahí acerca de lo que Hume decía que rápidamente preparó una segunda edición de su texto, con un pórtico que era una cita de Baco de Verulamio (o sea, Francis Bacon) que precisaba: "No hablemos de nosotros mismos". Y ello porque evidentemente Hume ponía su yo por delante en cada frase. Y eso es lo que hace Sterne: poner su yo (que es también el de Tristram o el de Yorick) delante de cada frase, para presentárnoslo como un proceso en el que va constituyéndose al "chocar" con el yo ya hecho y sólido de los demás personajes. Que precisamente por ello se nos presentan casi como arquetipos sin fisuras.

En una palabra: lo que podemos leer en el libro de Sterne es que el intento de inventarse la vida como "yo libre" resulta imposible, o al menos lleno de brechas y heridas, puesto que desde antes de nacer (como ocurre con Tristram niño o como ocurre con Don Quijote que nace a los cincuenta años) ya hay todo un mundo que nos espera (familiar en Tristram, extraño en Don Quijote) y que nos va a ir imponiendo sus signos, su lenguaje y su inconsciente vital y configurando nuestro interior hasta acabar en fracaso.

No teológicamente, porque la vida sea finita, o, al modo hegeliano, porque el Espíritu se extravíe (como diría Kojève), sino literalmente: porque no nacemos libres sino que el inconsciente de la supuesta libertad se nos impone y nos destroza.

5.- Y así llegamos a nuestro tercer estallido o contradicción literaria y artística: la invisibilidad de la mujer, en este terreno y en cualquier otro. Seré brevísimo porque es un trabajo que tengo en proceso.

Empecé a analizarlo a propósito de La obra maestra desconocida de Balzac ${ }^{5}$. Esta novela corta o "nouvelle" la publicó Balzac en I83I (en su primera versión) en dos partes en la revista L'Artiste. Luego se reeditó en I93I, con ilustraciones de Picasso. Lógico, pues el relato nos cuenta una historia de tres pintores discutiendo sobre la vida y el arte o cómo dar vida al arte. El protagonista aparente es el joven Poussin (el texto se sitúa a principios del XVII) junto a un pintor imaginario, Frenhofer y otro real, Pourbus "el joven". Frenhofer cuenta que está pintando una obra maestra sobre una mujer, su gran amor, a quien nunca veremos. Pero de nuevo debo matizar: la primera parte la titula Balzac Guillette y la segunda parte Catherine Lescault, la supuesta amante de Frenhofer. A su vez Gillette es la joven amante de Poussin, a la que este exige que pose desnuda para Frenhofer, algo que a ella la desconcierta por completo. En la segunda parte, todos están en casa de Frenhofer: este les va a mostrar su obra maestra. Pero al quitar el velo que tapa el cuadro, ellos sólo ven manchas borrosas e inconexas y apenas se distingue el pie desnudo de una mujer. Mientras tanto, Gillette está desnuda y acurrucada en un rincón, pero también invisible: sólo Poussin se da cuenta de que está allí. Aquella noche, cuando todos

5 Cfr. H. de Balzac, La obra maestra desconocida, trad. de Javier Albiñana, prólogo de Palau i Fabre, ilustraciones de Picasso, Círculo de lectores/ Galaxia Gutemberg, Barcelona, 2ooo. En el texto me remito a mi artículo: “Algo más sobre La obra maestra desconocida. (Y sobre algunas categorías críticas en Literatura y en Arte)", en: Diálogos de Arte. Homenaje al profesor Domingo Sánchez-Mesa Martín, Universidad de Granada, 2014, pp. 629-638. 
se han ido, Frenhofer quema su casa y sus cuadros y muere entre las llamas. He aquí pues un libro en el que la invisibilidad de la mujer es prácticamente absoluta. Lo sintomático es que Richard Hamilton", el artista británico creador del término "Pop” en I956, con su famosa imagen/ collage titulada "¿Qué es lo que hace que los hogares de hoy sean tan diferentes, tan atractivos?", culminara su larga trayectoria en 2OIO-2OII con tres paneles creados por ordenador y sobrepintados a mano (Hamilton había aprendido a pintar sobre el cristal gracias a su amigo y maestro Marcel Duchamp) cuyo título es precisamente "La obra maestra desconocida". Parece increíble, pero Hamilton incluye ahí el taller de un Poussin maduro y la sobreimpresión de los autorretratos de Tiziano, Courbet y del propio Poussin. Y una mujer desnuda tendida sobre la cama que sin duda evoca a la joven Gillette.

Ahora bien: ¿por qué Poussin, Tiziano y Courbet? Sencillamente porque ellos sí habían intentado mostrar de algún modo la visibilidad de la mujer: Poussin había pintado un paisaje con una imagen absolutamente insólita en i627. El desnudo de una mujer masturbándose en un claro del bosque, con un sátiro a sus pies y otro masturbándose a su vez tras un árbol. Tiziano porque había pintado la "Venus del perrito" o "Venus de Urbino" que posiblemente inspiró a Poussin (y desde luego a la Olimpia de Manet) o quizá por la mujer que aparece desnuda a la derecha en "El carnaval de los Andrios"; y Courbet por su cuadro "El origen del mundo", el célebre cuadro con el sexo femenino en primer plano, que Lacan guardó durante tantos años en su casa.

Lo decisivo en estos paneles de Hamilton no es obviamente que incluyera ahí a tres pintores de desnudos femeninos (tales desnudos son obviamente infinitos), sino que lo haga concentrándolo todo en la imagen de la mujer en primer plano y con tres célebres pintores observándola por fin. Y digo que esto es decisivo porque, en el texto de Balzac, el pintor Frenhofer había exclamado con rabia contra sus dos compañeros: “¡Pintáis a la mujer, pero no sabéis verla!". No se trata, pues, de pintar a la mujer sino de la cuestión ideológica absoluta de la invisibilidad de la mujer. En nuestra sociedad la mujer debería ser visible pero sin embargo aún no lo es.

Se suele decir -desde Marx- que Balzac bajó a los sótanos para dar nombre a los que no lo tienen, para hacer visible lo invisible. Pues bien, eso es algo obvio en La obra maestra desconocida. Sólo que curiosamente algo similar fue lo que intentó Jean-Luc Godard en r965 en la película Alphaville?.

En todas las películas de espías al estilo oo7, básicamente por tanto las de James Bond, las “chicas Bond” eran absolutamente visibles como desnudos pero invisibles como personas; al igual que en todas las películas de ciencia-ficción la sombra del "Gran Hermano" de la novela de Orwell 1984 , planeaba bajo el sonido de la voz de un robot tirano y las mujeres eran igualmente secundarias y robóticas. Pues bien: en Alphaville, Godard consigue que la mujer robótica se vaya transformando en una mujer auténticamente

\footnotetext{
${ }^{6}$ Cfr. Sobre Richard Hamilton el muy buen catálogo editado por el Museo Reina Sofía, Madrid, Junio-Octubre, 2OI4.

${ }^{7}$ Vid. Jean Luc Godard: Alphaville. Une Étrange Aventure de Lemmy Caution, 1965, 98 '.
} 
viva y visible. Claro que en gran parte gracias a Anna Karina (recién divorciada del director, aunque aún seguían viviendo juntos). De ella decía Godard: "Es una actriz nórdica, que tiene mucho en común con las actrices del cine mudo. Actúa con todo el cuerpo y no sólo de manera psicológica". En Alphaville, la ciudad del futuro (que en realidad eran los suburbios que entonces empezaron a proliferar en París) en todos los hoteles hay una especie de Biblia (como suele ocurrir en Estados Unidos), una biblia, que es de hecho un diccionario. En este Diccionario están prohibidas todas las palabras que puedan expresar sentimientos o emociones: por ejemplo, llorar o amar. Eddie Constantine (aquel mal actor americano que triunfaba en Francia como el detective Lemmy Caution) le ofrece a la protagonista un nuevo diccionario, para que ella pueda aprender a leer. No es sólo que Constantine ejerza en cierto modo de Pigmalión, es ella la que se esfuerza en ser distinta. ¿Y cuál es el Diccionario que ella lee? Pues algo decisivo: se trata del libro de poemas de Paul Éluard titulado Capital del dolor, donde se incluye el famosísimo poema "Libertad". Al final de la historia, cuando ambos huyen en coche de Alphaville, la protagonista ha aprendido ya a balbucear algo nuevo. Mira a Constantine y le dice "Te amo".

Así termina la película y así terminamos nosotros. Pero fijémonos: ese "te amo" no es un sentimentalismo romántico, siempre atribuido a la mujer en nuestras sociedades. Mucho más agudamente, Godard nos trata de mostrar el hecho de que bajo el peso insoportable de la competencia capitalista, que nos conduce a la soledad y al antagonismo, cualquier otro tipo de relaciones personales está desapareciendo o ha desaparecido ya.

Pues en realidad se trata de eso: de la lucha por encontrar un diccionario otro, un inconsciente otro, que nos permita alcanzar el sueño de la libertad sin explotación, donde lo invisible pueda ser visible. Quizá comenzando por reconocer la gran trampa de nuestros días -y ya desde el XVIII- la trampa del “yo-soy-libre-por naturaleza”, el falseamiento de la libertad que se nos ofrece. No es imposible luchar por la conquista de ese otro tipo de "libertad real". 